\title{
RecArd: Robô baseado na plataforma Arduino como facilitador no processo de ensino-aprendizagem multidisciplinar
}

\author{
João Lucas de Souza Silva, DEEL/IFBA, jlucas.silva@ifba.edu.br \\ Michelle Melo Cavalcante, DEEL/IFBA, michellemelo.c@ifba.edu.br \\ Fabiano Amorim Vaz, CIN/UFPE, fav2@cin.ufpe.br \\ Jamilson Ramalho Dantas, CIN/UFPE, jrd@cin.ufpe.br \\ Esdriane Cabral Viana, DEPEN/IFBA, esdriane@ifba.edu.br
}

\begin{abstract}
Resumo. O presente trabalho tem por finalidade auxiliar e complementar o ensino-aprendizagem de disciplinas nas áreas de computação, eletrônica $e$ mecânica com a utilização de um robô denominado RecArd. Na tentativa de contribuir para uma mudança por meio da viabilização de aulas práticas, utilizou-se a plataforma de prototipagem Arduino e através desta montou-se o robô controlado com um smartphone e com capacidade de desviar de obstáculos. A ferramenta apresentou potencialidades que são consideradas relevantes no processo de ensino-aprendizagem, principalmente, em áreas multidisciplinares. Com base nos experimentos e resultados, pode-se indicar que o uso da robótica na educação deve ser considerado como modelo reformulador de métodos de ensino.
\end{abstract}

Palavras-chaves. Robótica, Educação, RecArd, Arduino.

\section{RecArd: Robot based on the Arduino platform as a tool to assist in multidisciplinary teaching-learning process}

\begin{abstract}
This work has the aim assist and supplement the teaching-learning courses in the areas of computing, electronics and mechanics with the use of a robot named RecArd. In an attempt to contribute to change through facilitation of practical classes, used the Arduino prototyping platform and through this was set up controlled robot with a smartphone and the ability to avoid obstacles. The tool presented potentialities that are considered relevant in the teaching-learning process, especially in multidisciplinary areas. Based on the experiments and results, can indicate that the use of robotics in education should be considered a change in the teaching model.
\end{abstract}

Keywords. Robotics, Education, RecArd, Arduino.

\section{Introdução}

A robótica apresenta diversas aplicações para o homem contemporâneo, uma das mais conhecidas é a aplicação industrial, mas robôs podem ser usados para uma vasta gama de finalidades, como entretenimento: brinquedos, atores, monstros de filmes; realização de ações à distância, exploração de ambientes insalubres e, mais recentemente, para a educação.

No contexto de Robótica educacional, Prol (2007) define-o como a caracterização de ambientes de aprendizagem que reúnem materiais de sucata ou kits de montagem compostos por diversas peças, motores, sensores, controlados por um 
computador com software que permita programar o funcionamento dos modelos montados, dando ao aluno a oportunidade de desenvolver sua criatividade com a montagem de seu próprio modelo.

De acordo com Gardner (1995), no universo em que cada pessoa aprende de uma maneira diferente, de acordo com a sua inteligência específica, é necessário pregar o Construtivismo (Ackermann, 2001), desenvolvido por Piaget, onde o aprendizado deve ser adquirido a partir da relação do conhecimento com o cotidiano do aluno. Assim, aprender torna-se um processo mais intuitivo (Bastos et al., 2010).

Alinhado com a teoria construcionista defendida por Piaget, Parpet (2008) propõe a ideia de que os seres humanos aprendem melhor quando são envolvidos no planejamento e na construção dos objetos. Nessa circunstância, a Robótica Educacional ganha força por se tratar da aplicação da robótica na área pedagógica, com o objetivo de disponibilizar aos alunos a oportunidade de criar soluções voltadas ao mundo real, possibilitando o aprendizado de forma dinâmica e estimulante. Tal aplicação é capaz de unir atividades de mecânica, como, por exemplo, a construção dos objetos controlados e atividades de raciocínio lógico, que envolvem cálculos de circuitos eletrônicos e desenvolvimento de programas que visam fazer com que determinado circuito eletrônico cumpra as atividades especificadas. (Santos et al., 2010)

Desta forma, percebe-se o grande potencial da robótica educacional como ferramenta multidisciplinar, visto que, a construção de protótipos, em geral faz com que o aluno questione e seja capaz de relacionar diferentes conhecimentos e aptidões, de forma a solucionar um problema. A busca por soluções estimula o espírito investigativo, fortemente motivado pela curiosidade, e permite que o aluno extrapole os conhecimentos individuais de cada disciplina. Assim a robótica assume o papel de uma ponte de ligação interdisciplinar visando a construção do conhecimento coletivo através da aplicação com a realidade. (Santos et al., 2010)

Frente a este cenário, o presente trabalho montou o RecArd, que consiste em um carro-rôbo, a partir da plataforma de prototipagem Arduino, como um novo método para auxiliar e complementar o ensino-aprendizagem multidisciplinar. $\mathrm{O}$ trabalho mostrou aos discentes de forma mais ampla assuntos voltados para computação e eletrônica para que estes associassem a disciplina com o seu cotidiano. Para mais, o projeto pretende estimular o estudante a pensar, a aguçar a sua curiosidade científica e a perceber os benefícios da robótica educacional para o desenvolvimento e aprimoramento da sua capacidade de memorização, seu raciocínio, sua concentração e atenção.

O restante do trabalho está organizado como segue. A seção 2 apresenta os trabalhos relacionados, a seção 3 aborda uma visão geral sobre o Arduino e o RecArd, a seção 4 expõe os materiais e métodos utilizados no projeto, a seção 5 discute, relaciona a robótica educacional com o RecArd e apresenta os resultados do projeto e, por fim, a seção 6 apresenta as conclusões do projeto.

\section{Trabalhos relacionados}

Existem trabalhos com o intuito de melhorar o ensino-aprendizagem em várias áreas e disciplinas voltadas para programação, robótica, eletrotécnica, eletrônica, entre outras, 
através da Robótica educacional, como é o caso do Programa de Educação Tutorial da Engenharia Elétrica da Universidade Federal Fluminense (PET-Elétrica UFF).

Segundo Júnior et al. (2013), o programa PET-Elétrica da UFF desenvolve as aulas de Robótica Educacional Como Ferramenta de Aprendizado para o Ensino Médio com objetivo de trazer para estudantes de Ensino Médio atividades práticas que tracem paralelos entre disciplinas teóricas apresentadas no Ensino médio, como Física e Matemática com o uso dos kits Lego Mindstorms NXT. Com isso, os alunos poderão criar experimentos onde será posto em prática a teoria das salas de aulas. Além disso, os alunos terão a chance de se aproximar de certos assuntos que se tornam enormes fontes de aprendizado e diversão, como é o caso da Robótica e Programação.

O trabalho de Perez et al. (2013), inicia-se com a utilização do Arduino visando os cursos de eletrônica, programação e robótica para alunos do ensino médio da rede estadual de educação. O principal objetivo é capacitar os alunos do ensino médio a projetarem e desenvolverem pequenos dispositivos robóticos, bem como incentivar estes a ingressarem em cursos de engenharia.

O principal diferencial do presente trabalho em relação aos demais surge do intuito de combinar diversos conhecimentos como eletrônica, mecânica e programação para que os discentes aprendam não só os conteúdos que fazem partes de suas disciplinas curriculares, mas também, para mostrar-lhes a importância e a relevância dessas áreas e das disciplinas através da simples construção de um robô de baixo custo. Logo, o atual projeto do RecArd visa incentivar, motivar e responder dúvidas que os discentes possam apresentar ao desenvolver atividades práticas.

\section{Arduino e RecArd}

Este capítulo traz uma visão geral do projeto Arduino, sua história e informações sobre licença e funcionamento, além do RecArd, um robô criado com o intuito de demostrar a facilidade de se trabalhar com esse tipo de projeto e como forma de incentivar alunos do ensino técnico e superior.

\subsection{Arduino}

A plataforma Arduino surgiu em 2005, feito por Massimo Benzi, David Cuartielles, Tom Igoe, Gianluca Martino e David Mellis, com o objetivo de criar um dispositivo para controlar projetos construídos de uma forma mais acessível e simples do que outros sistemas disponíveis no mercado. (Silva et al., 2014)

A plataforma Arduino, é uma plataforma open-source baseada em hardware e software para as áreas de automação e robótica. Ou seja, pode-se utilizar comercialmente e pessoalmente, além de permitir obras derivadas. É possível adicionar na placa diversos tipos de componentes eletrônicos direcionados e programados para um determinado projeto. (Cavalcante et al., 2014)

O Arduino utiliza um Microcontrolador ATMEGA (chip controlador gravável) com a função de receber e entregar o fluxo de informações com o controle por software (Silva et al., 2014). A linguagem de programação utilizada é um conjunto de funções da linguagem $\mathrm{C} / \mathrm{C}++$ com algumas mudanças. A figura 1 ilustra o Arduino UNO R3. 


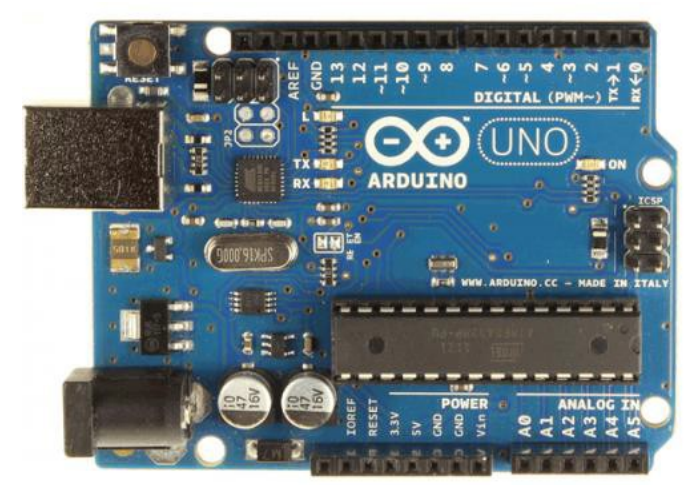

Figura 1 - Placa Arduino UNO R3.

\subsection{RecArd}

A construção do Rôbo surgiu no projeto de iniciação científica do presente autor intitulado por "RecArd: Um robô para auxílio na reconstrução tridimensional com aplicação em ambientes virtuais multissensorial”, cujo objetivo era utilizar o robô RecArd com uma câmera e/ou Kinect ${ }^{1}$ para capturar áreas e reconstruí-las tridimensionalmente. Ou seja, um processo de digitalização.

Entretanto, percebeu-se que o RecArd possuía capacidade para ser uma ótima ferramenta para o aprendizado de conceitos de eletrônica e computação, principalmente para desenvolver a lógica de programação, de maneira lúdica, interativa e de baixo custo. Assim, tem-se a tecnologia auxiliando no processo de ensino-aprendizagem, o que torna-o uma ferramenta essencial neste processo (Joly, 2002).

Para o processo educacional, o robô foi feito com base na plataforma Arduino e com uma comunicação bluetooth que possibilitou a conexão e o controle do mesmo através de um smartphone. Neste caso, adotou-se o Lumia 925 da Microsoft/Nokia, utilizando uma aplicação denominada "BT Terminal"”. O robô ainda possui um sensor ultrassônico para evitar colisões com objetos e obstáculos.

\section{Materiais e métodos}

A metodologia inicial empregada no projeto consistiu em uma pesquisa bibliográfica a partir dos autores Margolis (2011) e Mcroberts (2011) onde abordam e introduzem as propriedades e características do Arduino. Após o estudo bibliográfico, definiu-se os materiais para a montagem do robô e após montado, uma pesquisa exploratória ajudou a verificar os benefícios da inserção do Robô RecArd em salas de aula.

A pesquisa foi analisada por uma amostra de 34 estudantes do Curso Técnico de Informática e Eletromecânica, bem como de estudantes do ensino superior do Curso de Engenharia Elétrica e por 03 professores da área de exatas.

\footnotetext{
${ }^{1}$ Kinect: Dispositivo da Microsoft criado para melhorar a interação homem-máquina no console de jogos Xbox.

${ }^{2}$ BT Terminal: Aplicativo desenvolvido pela DevFor8, com objetivo de controlar dispositivos como Arduino através de um smartphone enviando comandos por uma terminal via bluetooth.
} 
Os materiais utilizados para construção do robô estão relacionados na Tabela 1. Além do Arduino, destaca-se o uso do Motor Shield L293d que serviu para possibilitar o uso dos motores DC (Motor de Corrente Contínua) para realizar o giro das rodas e também o Módulo Bluetooth RS232 HC-06, responsável pela comunicação wireless entre o smartphone (Lumia 925) e o RecArd.

Tabela 1 - Materiais para a elaboração RecArd.

\begin{tabular}{c|c}
\hline QUANTIDADE & MATERIAIS \\
\hline 01 & Placa Arduino UNO R3 \\
\hline 01 & Mini-Protoboard \\
\hline 04 & Motores DC com caixa de Redução \\
\hline 04 & Chassi composto por duas placas de Acrílico \\
\hline 01 & Shield Ardafruit L293D (Ponte H) \\
\hline 01 & Resistor de 330 $\Omega$ \\
\hline 01 & Módulo Bluetooth HC-06 \\
\hline 01 & Sensor Ultrassônico \\
\hline 01 & Jumpers \\
\hline 01 & Conjunto de 4 pilhas AA para alimentar Arduino \\
\hline 21 & Conjunto de 8 pilhas AA para alimentar motores \\
\hline 01 & Smartphone Nokia Lumia 925 \\
\hline 01 &
\end{tabular}

Na Figura 2 é apresentado de forma detalhada e através de quatro vistas, sendo elas a frontal, superior, lateral e inferior, respectivamente, as partes constituintes do robô RecArd com os respectivos materiais citados anteriormente.

É possível perceber com clareza na Figura 2-A o sensor ultrassônico e uma parte do conjunto pilhas AA para alimentar os motores. Na Figura 2-B evidencia-se o módulo bluetooth HC-06 e o LED amarelo. Posteriormente, na Figura 2-C percebe-se a placa Arduino, o Shield Ardafruit L293D e o conjunto de 4 pilhas AA para alimentar Arduino. Por último, na Figura 2-D é apresentado os motores DC com caixa de redução. 


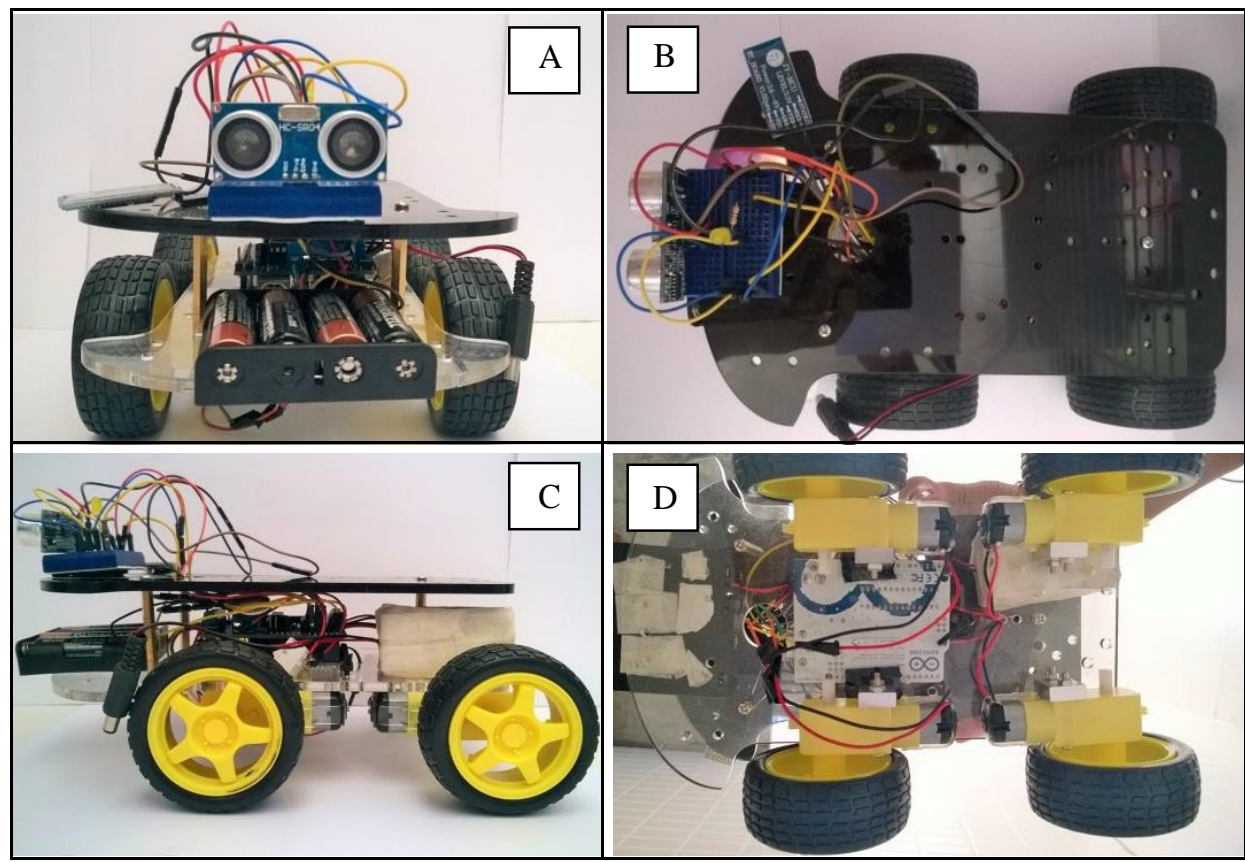

Figura 2 - Robô RecArd com vista: (A) Frontal, (B) Superior, (C) Lateral e (D) Inferior.

Na Figura 3 são apresentados o RecArd e o smartphone (Lumia 925). Na Figura 3-A o robô é ligado e pareado a conexão bluetooth com o módulo HC-06. Em seguida, na Figura 3-B, o aplicativo BT Terminal é aberto em sua tela de comandos. O comando "w" desloca o robô para frente, "s" desloca para trás, "a” para esquerda, "d" para direita e " $x$ " interrompe os motores. É válido destacar que a conexão do robô pode ser feita com outros modelos de smartphone, inclusive com outros sistemas, como o Android e iOS.

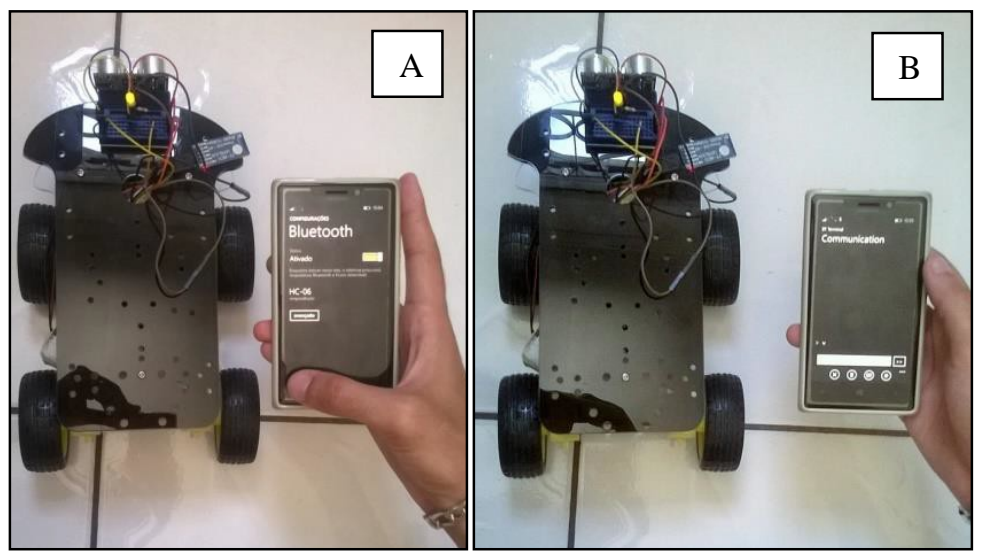

Figura 3 - Robô RecArd (A) pareando conexão bluetooth com Lumia 925 e (B) conectado com o BT Terminal.

Após a construção deste protótipo, foi iniciada a fase da análise do projeto. A análise foi realizada em duas etapas: A primeira consistiu na aplicação de questionários elaborados com respostas na escala nominal binária (perguntas diretas com respostas "Sim” ou "Não") respondidas somente pelos alunos. Na segunda parte, utilizou-se a Escala de Concordância de um a cinco, indicando péssimo e excelente, respectivamente. 


\section{Resultados e Discussão}

A presente seção aborda a inserção da robótica na educação bem como sua importância para, posteriormente, relatar e apresentar os resultados coletados com o projeto a partir das atuais pesquisas e debates em sala de aula.

\subsection{Robótica na Educação}

A robótica, por si, é notoriamente uma área que desperta bastante interesse nos indivíduos, bem como em pesquisadores de áreas transversais. O que buscou-se neste trabalho foi utilizar este potencial lúdico e multidisciplinar para desenvolver um mecanismo de auxílio no processo de ensino-aprendizagem.

Logo, o próprio desenvolvimento do robô veicular já despertava a curiosidade da comunidade acadêmica local, com questionamentos que abordavam diversos conteúdos curriculares nas áreas de Computação, Eletrônica, Mecânica e afins. Questões como: “O que são sensores e atuadores? Qual a diferença entre eles?”, "Por que se utiliza caixa de redução nos motores DC?”, “O que é e porque usar a Ponte H?”, “Por que usar um resistor acompanhado de um LED?”, “Como funciona o sensor ultrassônico?”, “Como funciona um módulo bluetooth?”, “Como é possível o RecArd obedecer aos comandos?”, “O que explica o uso de determinada voltagem para alimentar o robô?” e entre outros.

Portanto, resolveu-se utilizar o Robô como parte do processo de ensino, no anseio de investigar se o aprendizado seria facilitado. Os primeiros experimentos foram realizados com um grupo de alunos do Curso Técnico em Informática para ensinar linguagem de programação, mais especificamente, estruturas de decisão e repetição, bastante utilizadas na maioria dos softwares. Em seguida, os alunos de eletromecânica puderam praticar conceitos de mecânica ao entender o funcionamento do veículo controlado eletronicamente. Posteriormente, os experimentos se sucederam expandindose para outras disciplinas relacionadas.

\subsection{Análise dos Resultados}

Após a fase de experimentação, iniciou-se a análise dos dados levantados no experimento. Antes de ter contato com o RecArd, aos alunos-voluntários foi solicitado o preenchimento de um questionário que dispunha sobre a experiência prévia com robótica. Na Tabela 2 são apresentadas quatro questões elaboradas com respostas na escala nominal binária: respostas “Sim” ou "Não”.

Tabela 2 - Questionários para avaliação da Robótica Educacional aplicado aos estudantes.

\begin{tabular}{l|c|c|c}
\hline \multirow{2}{*}{ QUESTÕES } & \multicolumn{3}{|c}{ RESPOSTAS } \\
\cline { 2 - 4 } & SIM & NÃO & NÃO SEI \\
\hline \multirow{2}{*}{$\begin{array}{l}\text { 1. Você sabe o que é Robótica? } \\
\text { 2. Você já teve contato com Robótica } \\
\text { anteriormente? }\end{array}$} & $97,30 \%$ & $2,70 \%$ & - \\
\hline
\end{tabular}




\begin{tabular}{ll|l|l|l}
\hline $\begin{array}{l}\text { 3. Acha que a Robótica pode } \\
\text { contribuir/incentivar com a }\end{array}$ & $94,59 \%$ & $5,41 \%$ & $0,00 \%$ \\
$\begin{array}{l}\text { Educação? } \\
\text { 4. Você acredita que o Arduino pode } \\
\text { ajudar no ensino-aprendizagem de } \\
\text { matérias vistas em sala de aula? }\end{array}$ & $83,78 \%$ & $5,41 \%$ & $10,81 \%$ \\
\hline
\end{tabular}

Com a Tabela 2, observou-se que a maioria dos estudantes tinham uma noção básica sobre robótica. Entretanto, esta maioria (83,78\%), nunca teve contato direto com a robótica voltada para a educação do discente. Para os que conheciam a plataforma Arduino, estes tinham visto através de disciplinas do instituto de ensino em que estudavam e/ou internet.

Os valores da Tabela 2 indicam que os alunos, em sua grande maioria, consideraram a robótica como um estímulo de estudo e como um mecanismo positivo para auxiliar na didática dos professores. Entretanto, alguns estudantes tiveram receio quanto a implantação de métodos desse tipo, alegando que os professores podem criar expectativas ao prometer trazer um projeto do tipo e por algum motivo, a ideia não se concretizar. Além disso, foi mencionado que o projeto poderia levar os discentes a se distraírem, tirando o foco do ensino.

Na fase posterior, após a utilização em sala de aula, os alunos foram novamente instigados a preencherem o formulário, de forma voluntária. Este segundo questionário, mais sucinto, possuía como objetivo averiguar o nível de concordância e aceitação dos estudantes sobre a implantação do projeto.

Por fim, a Figura 4 ilustra o resultado da avaliação do projeto na escala de concordância (Escala Likert) com 5 notas no formato ordinal: (5) Excelente, (4) Bom, (3) Regular, (2) Ruim e (1) Péssimo, avaliados pelos professores e alunos presentes durante a aplicação do projeto.

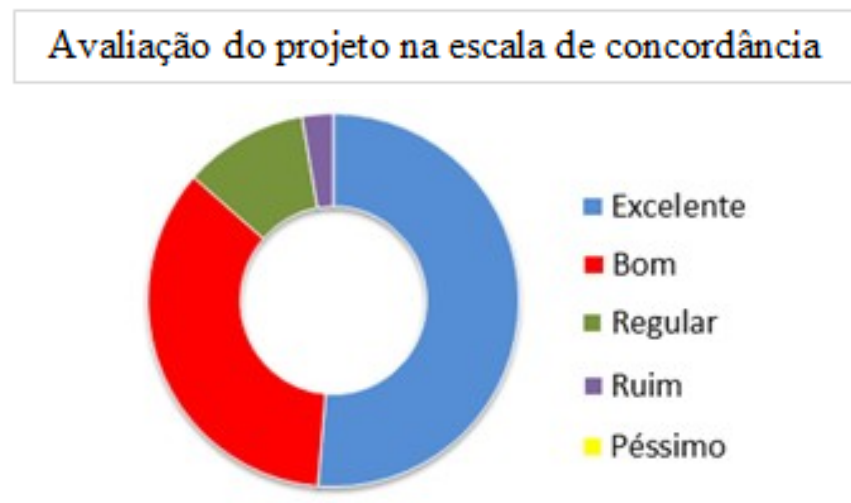

Figura 4 - Resultados na escala de concordância para o projeto

Os resultados obtidos na Figura 4 reforçam os resultados apresentados nos questionários da Tabela 2, onde 51,35\% (19 pessoas) dos participantes atribuíram a pontuação máxima ao projeto, 35,14\% (13 pessoas) nota quatro, 10,81\% (4 pessoas) nota três e $2,70 \%$ (1 pessoa) atribuíram nota dois. 
Para o participante que considerou o projeto ruim, este afirmou que um projeto desse tipo pode agitar os discentes a ponto de distraí-los, pois é algo novo e o excesso de vontade pode atrapalhar e deixar passar determinadas informações.

Destaca-se também que foram obtidas várias opiniões, tanto de professores como alunos, para melhorar o projeto. Dentre as opiniões, salienta-se a sugestão de um estudante de ensinar simulações de sistemas eletro-energéticos e controle de ambientes para alunos de graduação em engenharia elétrica. Outro aluno mencionou que o projeto diminui a distância entre o estudante e a tecnologia, além de diminuir a abstração de conteúdos que existe em algumas disciplinas.

\section{Considerações finais}

As habilidades e competências acrescidas com o projeto permitem que os alunos compreendam melhor as disciplinas nas áreas de eletrônica e computação principalmente, e, assim, obtenham um melhor desempenho nas mesmas. O estudante passa então a sentir um estímulo maior na busca do conhecimento quando conhece a aplicação prática de determinados conteúdo, isto é, o estudo une a teoria e a prática e assim faz com que dúvidas sejam solucionadas de forma mais fácil, além de fazer com que o aluno aprenda de uma melhor forma a união do hardware e software e do uso dos componentes eletrônicos.

Como ponto negativo referente ao projeto cita-se, principalmente, a dificuldade em adquirir alguns materiais e equipamentos eletrônicos, visto que só é possível encontrar estes com facilidade via internet.

Como trabalhos futuros, pretende-se aprimorar o robô e deixá-lo mais interativo, incluindo a ele outros sensores e equipamentos, ampliando também o tempo de uso do robô na sala de aula. Com isso, planeja-se ministrar palestras e minicursos para divulgar o trabalho e para estender o conhecimento e atividades multidisciplinares. Em paralelo, pretende-se concluir o objetivo inicial do robô: reconstrução tridimensional para produção e gerenciamento de ambientes virtuais.

\section{Agradecimentos}

Os autores agradecem ao Instituto Federal de Educação, Ciência e Tecnologia da Bahia (IFBA), Campus Paulo Afonso, pelo apoio técnico e à Fundação de Amparo à Pesquisa do Estado da Bahia (FAPESB) pelo apoio financeiro através do Programa de Bolsas de Iniciação Científica (PIBIC/IFBA).

\section{Referências}

\section{ACKERMAN, E. Piaget's Construtivism, Parpert's Constructionism: What's} the difference?. MIT Media Lab, 2001.

BASTOS, B.L. et al. Schatch, Arduino e o Construcionismo: Ferramentas para a educação. In: Seminário de Tecnologia Educacional de Araucária, 2010, Araucária. Anais. Araucária: Secretaria Municipal de Educação de Araucária, 2010. 
CAVALCANTE, M.M. et al. A Plataforma Arduino pra fins didáticos: Estudo de caso com recolhimento de dados a partir do PLX-DAQ. In: Congresso da Sociedade Brasileira de Computação, 2014, Brasília. Anais. Brasília: Sociedade Brasileira de Computação, p.1655-1664.

GARDNER, H. Inteligências Múltiplas. Artes Médicas, Porto Alegre, 1995.

JOLY, M.C.R.A. Tecnologia no ensino: implicações para a aprendizagem. São Paulo: Casa do Psicólogo.

JÚNIOR, E.A.N. et al. Apostila de Robótica Educacional. Programa de Educação Tutorial da Engenharia Elétrica - PET-Elétrica. Universidade Federal Fluminense (UFF). Niterói, RJ. 2013.

MARGOLIS, M. Arduino Cookbook. Sebastopol: O’Reilly Media, 2011.

MCROBERTS, M. Arduino básico. São Paulo: Novatec Apress, 2011.

PARPET, S. A máquina das crianças: repensando a escola na era da informática. Tradução de Sandra Costa. Porto Alegre: Artes Médicas, 2008. p.210.

PEREZ, A.L.F. et al., 2013. Uso da Plataforma Arduino para o Ensino e o Aprendizado de Robótica. In: International Conference on Interactive Computer aided Blended Learning, 2013.

PROL, L.C.A. Diferentes materiais para uso na robótica educacional: A diversidade que pode promover o desenvolvimento de diferentes competências e habilidades. In: Marcusso, N.; Brito, P.; Telles, M. A Tecnologia Transformando a Educação: Casos de Aplicação. São Paulo: Praxis, 2007. p.133-139.

SANTOS, F.L. et al. A Robótica Educacional como Abordagem de Baixo Custo para o Ensino de Computação em Cursos Técnicos e Tecnológicos. In: Congresso da Sociedade Brasileira de Computação, 2010, Belo Horizonte. Anais. Belo Horizonte: Sociedade Brasileira de Computação, p.1304-1313.

SILVA, J.L.S. et al. Plataforma Arduino integrado ao PLX-DAQ: Análise e aprimoramento de sensores com ênfase no LM35. In: Escola Regional de Computação Bahia, Alagoas e Sergipe. Feira de Santana. Anais. Feira de Santana: Sociedade Brasileira de Computação, 2014. 